\title{
Nonlinear response to electric field in extended Hubbard models
}

\author{
D. Nasr Esfahani, ${ }^{*}$ L. Covaci, ${ }^{\dagger}$ and F. M. Peeters ${ }^{\ddagger}$ \\ Departement Fysica, Universiteit Antwerpen, Groenenborgerlaan 171, B-2020 Antwerpen, Belgium \\ (Received 14 April 2014; revised manuscript received 7 October 2014; published 14 November 2014)
}

\begin{abstract}
The electric-field response of a one-dimensional ring of interacting fermions, where the interactions are described by the extended Hubbard model, is investigated. By using an accurate real-time propagation scheme based on the Chebyshev expansion of the evolution operator, we uncover various nonlinear regimes for a range of interaction parameters that allows modeling of metallic and insulating (either charge density wave or spin density wave insulators) rings. The metallic regime appears at the phase boundary between the two insulating phases and provides the opportunity to describe either weakly or strongly correlated metals. We find that the fidelity susceptibility of the ground state as a function of magnetic flux piercing the ring provides a very good measure of the short-time response. Even completely different interacting regimes behave in a similar manner at short time scales as long as the ground-state fidelity susceptibility is the same. Depending on the strength of the electric field we find various types of responses: persistent currents in the insulating phase, a dissipative regime, or damped Bloch-like oscillations with varying frequencies or even irregular in nature. Furthermore, we also consider the dimerization of the ring and describe the response of a correlated band insulator. In this case the distribution of the energy levels is more clustered and the Bloch-like oscillations become even more irregular.
\end{abstract}

DOI: 10.1103/PhysRevB.90.205121

PACS number(s): 71.30.+h, 71.27.+a

\section{INTRODUCTION}

The investigation of real-time dynamics of a closed system consisting of interacting particles is important not only for the evaluation of experimentally relevant quantities, but also supplies reliable information about the general properties of the Hamiltonian as long as one measures an appropriate set of observables throughout the propagation process [1]. This is of particular interest when the dimension of the Hilbert space is very large in which case it is not possible to access the whole energy spectrum.

There exist several approaches for solving the problem of real-time propagation of closed interacting systems. Among them are the numerically exact polynomial expansions [2,3] or the approximate Lanczos propagation method [4], the state-ofthe-art time-dependent density matrix renormalization group (tDMRG) [5], and nonequilibrium dynamical mean field theory (nDMFT) [6,7]. Among the others are the time-dependent numerical renormalization group [8], unitary perturbation theory [9], nonequilibrium Monte Carlo schemes [10], the real-time-dependent renormalization group scheme [11], and transfer matrix approaches [12].

The common thread for all these methods is that it is not necessary to access the whole spectrum in order to evaluate time-dependent expectation values; hence this makes it feasible to investigate a large class of interacting systems.

The Hubbard Hamiltonian, as a standard model for interacting lattice fermions, has been the central subject of numerous theoretical investigations, part of which focused on real-time quench dynamics [13-17], real-time studies based on the relaxation dynamics of specifically prepared excited states [18], and the effect of an external electric field [6,19-26].

\footnotetext{
*Davoud.NasrEsfahani@uantwerpen.be

†lucian@covaci.org

†Francois.Peeters@uantwerpen.be
}

As an important time-dependent phenomenon, the electric breakdown of a one-dimensional Mott insulator has been theoretically investigated $[27,28]$ and the analysis was based on a Landau-Zener (LZ) [29,30] mechanism, which showed an exponential decay of the overlap with the initial ground state as function of time, for short time scales. The exponent of the decay rate is proportional to the square of the charge gap of the system [28]; however this is not universal and this quadratic dependence could deviate in specific cases. The later observation could be assigned to the fact that either the two-level approximation or the tendency of the propagated state to overlap only with the first excited state could be violated in some cases. We found that, similarly to Ref. [31], the propagated state could have significant overlap with several higher excited states.

In order to alleviate these discrepancies of the two-level approximation we employ the recently proposed fidelity susceptibility [32,33] as a measure of the change in basis set when an external field is varied. This quantity is unbiased and can be calculated numerically exactly. Throughout this work we use it as a basis for comparing the short-time response of different insulating systems subjected to a constant electric field.

Beyond the short-time-scale ground-state decay, a question that grasped attention recently was focused on the dependence of the long-time electric field response on the ground-state properties and/or interaction parameters. A notable phenomenon that definitely depends on longer time scales and is beyond the ground-state decay mechanism based on the LZ tunneling is the appearance of Bloch oscillations (BOs). The existence of these oscillations has already been proven experimentally in semiconductor superlattices [34-37].

The damping of Bloch oscillations in a closed interacting fermionic system subjected to a uniform electric field has been described theoretically within the Falikov-Kimbal model [6] and the one-dimensional interacting spinless model [38], where it is shown that an integrable system subjected to a weak uniform field exhibits current oscillations with frequencies 
larger than the frequency of normal BOs. The damping of the BOs was further investigated within the one-dimensional Holstein model [39] and the infinite-dimensional Hubbard model [22].

By using an extended Hubbard model, one has the opportunity to design the interaction parameters in order to have a better understanding about the mechanism of the breakdown in short time scales and the appearance of BOs in longer time scales. The main aim of this paper is to investigate the differences between the nonlinear response of different kinds of closed systems of interacting fermions both in the insulating and the metallic regimes. This goal is achieved by employing a real-time propagation scheme together with the ground-state and spectral analysis.

Notice here that from the experimental point of view, the special case of electric breakdown of 1D Mott insulators has been realized experimentally either with a strong electric field $[40,41]$ or through photoinduced metal-insulator transitions in pump-probe experiments $[42,43]$. Further interest was recently triggered by the realization of fermionic optical lattice experiments, where the effect of an electric field on systems with designed interactions could be measured [44-47].

This paper is organized as follows: In Sec. II we present our model under study together with a brief description of the theoretical and numerical schemes. In Sec. III A we present our analysis of the response to constant electric field for a system of weakly interacting fermions, while in Sec. III B we perform the same study but for strongly interacting fermions. Finally, in Sec. IV we give our conclusions.

\section{MODEL AND METHOD}

Our model under investigation is a one-dimensional closed system of interacting charged fermions with periodic boundary conditions. It can be described in the second-quantization formalism by an extended Hubbard model as follows:

$$
\begin{aligned}
\hat{H}= & -\sum_{i \sigma}\left[h_{i+1, i}(t) \hat{c}_{i+1 \sigma}^{\dagger} \hat{c}_{i \sigma}+\text { H.c. }\right]+\sum_{i} V \hat{n}_{i} \hat{n}_{i+1} \\
& +\sum_{i} U \hat{n}_{i \uparrow} \hat{n}_{i \downarrow} ;
\end{aligned}
$$

$\hat{c}_{i \sigma}^{\dagger}\left(\hat{c}_{i \sigma}\right)$ is the creation (annihilation) operator of a fermion at site $i$ and spin $\sigma=(\uparrow, \downarrow)$. The fermion density is defined as usual as $\hat{n}_{i}=\hat{n}_{i \uparrow}+\hat{n}_{i \downarrow}$ with $\hat{n}_{i \sigma}=\hat{c}_{i \sigma}^{\dagger} \hat{c}_{i \sigma}$. The first term in Eq. (1) represents the kinetic energy, where the hopping amplitude is taken to be time-dependent and by using the Peierls substitution becomes $h_{i+1, i}(t)=h_{i+1, i} e^{\frac{i e}{\hbar} \phi(t)}$ with $h_{i, i+1}=\left[h_{0}+(-1)^{i} \eta\right] . \phi=\phi_{\text {tot }} / L$ is the total magnetic flux piercing the ring divided by the number of sites and $\eta$ models a dimerization term. Hereafter we consider $\hbar=e=a=1$, where $a$ is the lattice constant and $e$ is the charge of particles. Interactions are either local between fermions with opposite spins, described by $U$, or nonlocal between fermions sitting on neighboring sites, described by $V$. All of the coupling constants which are reported in the following are scaled with $h_{0}=1$.

Throughout this work we consider an electric field, which is given by the time derivative of the flux, $\tilde{F}=-\dot{\phi}(t) / a$, in units of $h_{0} / e a$. In the following, the time coordinate is reported in units of $\hbar / h_{0}$, and all of the cases we consider are at half filling with $N_{\uparrow}=N_{\downarrow}=L / 2$, where $N_{\uparrow}$ and $N_{\downarrow}$ are the total number of spin-up and spin-down particles, respectively, and $L$ is the total number of sites.

Starting from parameters at time, $t=0$, we find the ground state of the resulting Hamiltonian and propagate it while considering the change of the coupling parameters as a function of time. To find the solution of the time-dependent Schrödinger equation

$$
H(\phi(t))|\psi(t)\rangle=i|\dot{\psi}(t)\rangle,
$$

where $|\dot{X}(t)\rangle=\partial|X(t)\rangle / \partial t$, one may write the solution as a superposition of the instantaneous eigenstates of the timedependent Hamiltonian as

$$
|\psi(t)\rangle=\sum_{n} c_{n}(t)|n(\phi(t))\rangle
$$

where $|n(\phi(t))\rangle$ are the instantaneous eigenstates of $\hat{H}(\phi(t))$, with

$$
H(\phi(t))|n(\phi(t))\rangle=E_{n}(\phi(t))|n(\phi(t))\rangle .
$$

By substituting $|\psi(t)\rangle$ as expressed by Eq. (3) into the Schrödinger equation and by using the change of variables as $\tilde{c}_{n}(t)=c_{n}(t) e^{i \theta_{n}(t)}$, with

$$
\theta_{n}(t)=\int_{0}^{t} E_{n}(\phi(\tau)) d \tau-i \int_{0}^{t}\langle n(\phi(\tau)) \mid \dot{n}(\phi(\tau))\rangle d \tau,
$$

one obtains the following set of coupled differential equations for the coefficients $\tilde{c}_{n}(t)$ :

$$
\dot{\tilde{c}}_{n}(t)=-\sum_{m \neq n} e^{i \theta_{n m}(t)} \tilde{c}_{m}(t)\langle n(\phi(t)) \mid \dot{m}(\phi(t))\rangle,
$$

where $\theta_{n m}(t)=\theta_{n}(t)-\theta_{m}(t)$. The change of basis set as a function of time manifests itself in the $\langle n(\phi(t)) \mid \dot{m}(\phi(t))\rangle$ term on the right-hand side of Eq. (6). By starting from the ground state of the Hamiltonian at $t=0\left[\left|\psi_{0}(0)\right\rangle\right]$, i.e., setting $\left|c_{0}(0)\right|=1$ and $\left|c_{n}(0)\right|=0$ for $n \neq 0$ in Eq. (3), and as long as the terms $\left\langle\psi_{0}(\phi(t)) \mid \dot{n}(\phi(t))\right\rangle \simeq 0$ during the evolution, then one arrives at the adiabatic regime where $|\psi(t)\rangle$ only follows the ground state of the instantaneous Hamiltonian such that $\left|c_{0}(t)\right| \sim 1$ and $\left|c_{n}(t)\right| \sim 0$ for $n \neq 0$. Moreover, during the adiabatic time evolution, the coefficient $c_{0}(t)$ only acquires a phase that is a combination of the geometrical (Berry) [48] and the dynamical phases. Moreover, it is possible to express terms $\left\langle\psi_{0}(\phi(t)) \mid \dot{n}(\phi(t))\right\rangle$ as

$$
\left\langle\psi_{0}(\phi(t)) \mid \dot{n}(\phi(t))\right\rangle=\dot{\phi} \times \frac{\left\langle\psi_{0}(\phi(t))\left|\hat{J}_{\mathrm{tot}}(\phi(t))\right| n(\phi(t))\right\rangle}{E_{n}(\phi(t))-E_{0}(\phi(t))},
$$

where $\hat{J}_{\text {tot }}(\phi(t))=\partial_{\phi} H(\phi(t))$. As is obvious from the above expression the second term on the right-hand side of Eq. (7) does not have any explicit dependence on $\dot{\phi}$ and it is an intrinsic property of the system. For the nonadiabatic regime when $\left\langle\psi_{0}(\phi(t)) \mid \dot{n}(\phi(t))\right\rangle$ is large, Eq. (6) not only ensures the change in the magnitude of $c_{n}(t)$ but each coefficient further accumulates a complicated phase consisting of dynamical and Berry phases produced by the other states.

If we consider the ground state as the starting state for the time evolution, the quantity that measures the change of basis 
set as function of the external parameter $\phi$ is the ground-state fidelity [49], which is defined as

$$
\Xi(\phi)=\left|\left\langle\psi_{0}(\phi) \mid \psi_{0}(\phi+\delta \phi)\right\rangle\right| .
$$

It is possible to see that there is a close relationship between the ground-state fidelity and the coefficients that appear on the right-hand side of Eq. (7). The change in the ground-state wave function under an infinitesimal change of flux can be written as

$$
\begin{aligned}
\left|\psi_{0}(\phi+\delta \phi)\right\rangle= & \Lambda\left[\left|\psi_{0}(\phi)\right\rangle\right. \\
& \left.+\delta \phi \sum_{n \neq 0} \frac{\left\langle n(\phi)\left|\hat{J}_{\mathrm{tot}}(\phi)\right| \psi_{0}(\phi)\right\rangle}{E_{0}(\phi)-E_{n}(\phi)}|n(\phi)\rangle\right],
\end{aligned}
$$

where $\Lambda$ is a normalization factor. After normalization and considering $\delta \phi \ll 1$ one obtains that

$$
\left|\left\langle\psi_{0}(\phi) \mid \psi_{0}(\phi+\delta \phi)\right\rangle\right|^{2}=1-(\delta \phi)^{2} \chi_{\Xi}(\phi),
$$

where $\chi_{\Xi}(\phi)$ is the ground-state fidelity susceptibility which is defined as $[32,33,50]$

$$
\chi_{\Xi}(\phi)=\frac{1-\Xi^{2}(\phi)}{(\delta \phi)^{2}}=\sum_{n \neq 0} \frac{\left|\left\langle n(\phi)\left|\hat{J}_{\mathrm{tot}}(\phi)\right| \psi_{0}(\phi)\right\rangle\right|^{2}}{\left[E_{0}(\phi)-E_{n}(\phi)\right]^{2}} .
$$

The leading term in the fidelity expansion is of the order of $\delta \phi^{2}$. When comparing the terms on the right-hand side of Eq. (11) with terms that appear on the right-hand side of Eq. (6) [i.e., $\left\langle\psi_{0}(\phi(t)) \mid \dot{n}(\phi(t))\right\rangle$ terms] and by considering Eq. (7), one may infer that a larger $\chi_{\Xi}(\phi)$ leads to an enhanced nonadiabatic character of the time evolution when the system is driven by an external electric field.

In the following sections we will use the ground-state fidelity susceptibility as a basis for the comparison of the short-time response for different kinds of interactions modeled by Eq. (1). We do this in particular when the system is subjected to a constant and uniform electric field.

Although the instantaneous eigenstate representation of the time-dependent Schrödinger equation is very insightful, the solution of Eq. (6) is either very difficult or outright impossible to obtain for systems where the Hilbert space is very large since having the eigenstates at each moment is computationally very expensive. For the case of interacting fermions with spin the dimension of the Hilbert space for a small system, which consists of only 10 sites at half filling, is 63504 ( $N_{\uparrow}=N_{\downarrow}=5$ ) and to solve Eq. (6) is very difficult.

An alternative way to deal with the time-dependent Schrödinger equation is to exploit the form of the unitary time evolution operator

$$
\hat{U}(t)=\mathcal{T} e^{-i \int_{0}^{t_{f}} \hat{H}(\phi(\tau)) d \tau} \simeq \prod_{k}^{N} e^{-i \hat{H}\left(\phi\left(t_{k}+\delta t / 2\right)\right) \delta t},
$$

with $\delta t=t_{f} / N$, where $N$ is the number of steps, $t_{f}$ is the final time, $t_{k+1}=t_{k}+\delta t$, and $t_{0}=0$. Therefore, the problem is reduced to a stepwise change of the Hamiltonian and relaxation of the system with a time step equal to $\delta t$. Over each time step the Hamiltonian is considered to be time-independent and the relaxation of the wave function can be easily performed, by using the Chebyshev propagation method [2,3], which considers an expansion of the evolution operator in terms of orthogonal Chebyshev polynomials.

The wave function at $t_{k+1}$ can now be written as

$$
\begin{aligned}
\left|\psi\left(t_{k+1}\right)\right\rangle= & e^{-i b \delta t}\left[J_{0}(a \delta t) I+\sum_{s=1}^{\infty} 2(-i)^{s} J_{s}(a \delta t) T_{s}\right. \\
& \left.\times\left(\tilde{H}\left(\phi\left(t_{k}+\delta t / 2\right)\right)\right)\right]\left|\psi\left(t_{k}\right)\right\rangle,
\end{aligned}
$$

where $\tilde{H}=(\hat{H}-b I) / a, I$ is the unit matrix with the same dimension of $\hat{H}, b=\left(E_{\max }+E_{\min }\right) / 2$, and $a=\left(E_{\max }-\right.$ $\left.E_{\min }\right) /(2-\epsilon)$, where $E_{\max }$ and $E_{\min }$ are maximum and minimum eigenvalues of the Hamiltonian, which could be calculated by means of the Lanczos method [51] at the beginning of the time evolution. $J_{s}$ is the $s$ th-order Bessel function of the first kind and $T_{s}(x)$ is the Chebyshev polynomials which obey the recursion relation, $T_{s}(x)=2 x T_{s-1}(x)-T_{s-2}(x) . \epsilon$ is introduced in order to make sure that the absolute value of the extreme eigenvalues of $\tilde{H}$ is less than 1 . This is crucial for the Chebyshev method because the arguments of Chebyshev polynomials accept only values in the interval $[-1,1]$; moreover, notice here that choosing an appropriate $\epsilon$ prevents the calculation of $E_{\max }$ and $E_{\min }$ at each step of propagation. We truncate the series in Eq. (13) such that the propagated wave function becomes normalized up to machine accuracy in order to reduce error accumulation during the stepwise propagations. Moreover this also ensures that the propagation operator is unitary up to machine accuracy.

Having the wave function at each time step, then the coefficients from Eq. (3), $c_{n}(t)=\langle n(\phi(t)) \mid \psi(t)\rangle$, could be calculated for analysis purposes only whenever it is necessarily or possible to do so. In order to have some insight about the nature of the wave function, $|\psi(t)\rangle$, we further calculate the structure factors that are defined as

$$
C_{X}(q)=\frac{2}{L^{2}} \sum_{i=1}^{L} \sum_{j=1}^{L / 2} e^{i q r_{i, i+j}} \bar{X}_{i, i+j}
$$

where $\bar{X}_{s, k}=\left\langle\hat{X}_{s} \hat{X}_{k}\right\rangle-\left\langle\hat{X}_{s}\right\rangle\left\langle\hat{X}_{k}\right\rangle, s$ and $k$ are the site indexes (summation over $L / 2$ for $j$ is introduced because $\bar{X}_{s, k}$ is symmetric around $\bar{X}_{s, s+L / 2}$ due to the periodic boundary condition we considered), and $r_{s, k}$ is the distance between site $s$ and site $k$. We report spin density wave (SDW) order parameter $O_{\text {SDW }}=C_{\hat{S}}(\pi)$ with $\hat{S}_{s}=1 / 2\left(\hat{n}_{s \uparrow}-\hat{n}_{s \downarrow}\right)$ and charge density wave $(\mathrm{CDW})$ as $O_{\mathrm{CDW}}=C_{\hat{n}}(\pi)$, where $\hat{n}_{s}=\hat{n}_{s \uparrow}+\hat{n}_{s \downarrow}$ is the local density operator. We also report the value of the current as a function of time, which is defined as the expectation value of the current operator, $\hat{J}=\frac{i}{L} \sum_{s \sigma}\left[h_{s+1, s}(t) \hat{c}_{s+1 \sigma}^{\dagger} \hat{c}_{s \sigma}-\right.$ H.c. $]$.

\section{RESULTS}

In the following we set $h_{0}=1$ and all the coupling constants are scaled with $h_{0}$. Moreover we define the uniform electric field, $\tilde{F}$, as $\phi(t)=-\tilde{F} t$. For the sake of simplicity we define $F=\tilde{F} / 2 \pi$. We consider the time steps to be $\delta t=0.005$. We have tested all the results against a finer time grid in order to ensure that there is no quantitative difference over the parameter range considered here; moreover in the following, 
the dimerization parameter is set to $\eta=0$ except when otherwise stated.

Notice here that in the calculations that are reported here total particle number and the number of particles with specific spin are fixed explicitly. However, the Hamiltonian that is presented in Eq. (1) is translationally invariant, which means that the translation operator $\hat{T}(a)$, where $a$ is the lattice constant, commutes with the Hamiltonian. By exploiting this property the Hilbert space of the full Hamiltonian could be reduced to $L$ different blocks. The symmetrization of the basis set can be easily performed by applying the projector $\hat{P}_{k}=1 / L \sum_{j=0}^{L-1} e^{2 \pi \imath j k / L} \hat{T}^{j}(a)$, on the unsymmetrized basis sets $|\alpha\rangle$. The basis sets with different $k=0 \ldots L-1$ values do not mix and when they are used for the calculation of matrix elements of the Hamiltonian, we find that $\left\langle\alpha, k^{\prime}|\hat{H}| \alpha, k\right\rangle=0$ for $k \neq k^{\prime}$ and $|\alpha, k\rangle=\hat{P}_{k}|\alpha\rangle$. For a detailed description of the symmetrization of the basis sets we refer the reader to Ref. [52].

Clearly, the perturbation which is induced by the presence of $\phi$ as an external parameter does not break this translational symmetry; therefore this property could lead to the presence of crossings in the energy dispersion of different energy levels as function of $\phi$ due to the presence of extra symmetries. In other words, the ground state of the full Hamiltonian could belong to different symmetry blocks when the parameter $\phi$ is changed. However, because the change of $\phi$ does not mix states that belong to different blocks, one should only focus on the ground state $\chi_{\Xi}(\phi)$ within a specific block, here the block containing the initial ground-state wave function at $\phi=0$.

We start by showing in Fig. 1 the ground-state fidelity susceptibility, $\chi_{\Xi}(\phi)$ (for the block $k=0$ ), at $\phi=0.1 \pi$ for a system consisting of 10 sites at half filling for different values of $U$ and as a function of $V$. As is clear from the inset of Fig. 1, $\chi_{\Xi}(\phi)$ acquires the largest value at $\phi_{\text {anti }}=0.1 \pi$,

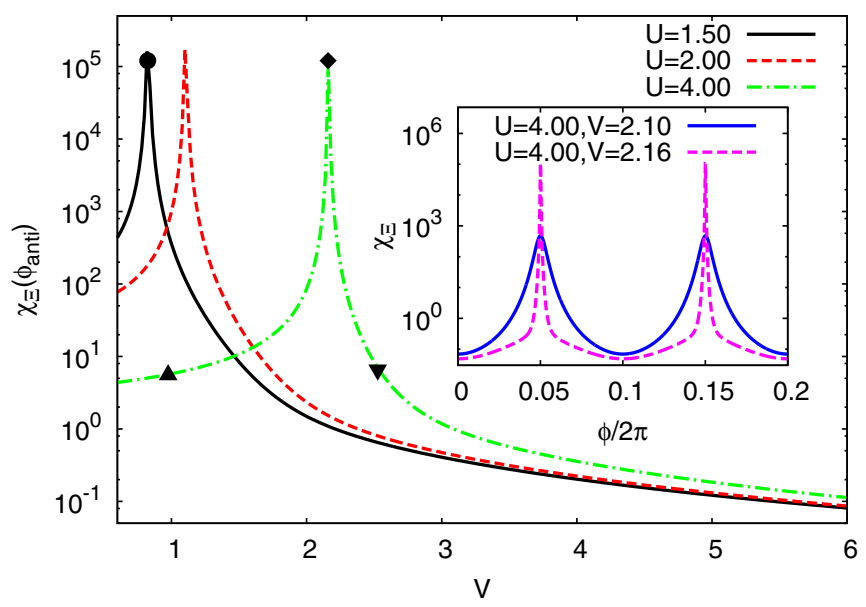

FIG. 1. (Color online) Ground-state fidelity susceptibility for a ring with $L=10$ and $N_{\uparrow}=N_{\downarrow}=5$ at $\phi_{\text {anti }}=0.1 \pi$ as a function of interactions. The inset shows the ground-state fidelity susceptibility as a function of $\phi / 2 \pi$ for different sets of parameters. The points represent specifically chosen pairs of parameters $U, V$ in order to model a weakly interacting metal (circle), a SDW insulator (triangle), a CDW insulator (upside down triangle), and a strongly interacting metal (diamond). which is an anticrossing point between the ground state and an excited state. Here we calculate $\chi_{\Xi}(\phi)$ numerically exactly with the use of the Lanczos [51] method and the differential form of $\chi_{\Xi}(\phi)$, i.e., $\chi_{\Xi}(\phi)=\left[1-\Xi^{2}(\phi)\right] / \delta \phi^{2}$, introduced in Eq. (11), and we use $\delta \phi=10^{-3}$ for the calculations presented in Fig. 1.

Notice that the susceptibility is largest, almost diverging, at specific values of $V$ for each $U$, whenever the relation $U \simeq 2 V$ is satisfied. This relation represents the boundary which separates the SDW and CDW phases [53], and was obtained within the DMRG approach for 1D chains of larger sizes. However, it is obvious that $\chi_{\Xi}\left(\phi_{\text {anti }}\right)$ can provide a good estimate on the location of the SDW-CDW phase boundary, although it does not provide any information about the details of the wave function (whether it describes SDW or CDW).

In order to compare the nonlinear response of different kinds of interacting systems we analyze different sets of interaction and hopping parameters. In particular we study three different cases: first we consider a system with $U=1.5$ and $V=0.82$, marked with a circle in Fig. 1, which shows an almost diverging $\chi_{\Xi}\left(\phi_{\text {anti }}\right)$ and has a vanishingly small charge gap, $\Delta_{\text {charge }}\left(\phi_{\text {anti }}\right) \simeq 6 \times 10^{-3}$, and therefore could be considered as a weakly interacting metal. Second, we use a dimerization parameter $\eta=0.4$, which opens up a gap $\left[\Delta_{\text {charge }}\left(\phi_{\text {anti }}\right)=1.74\right]$ and the system behaves as a correlated band insulator (BI). Finally, we choose a stronger interacting system with $U=4.0$ and three different values of $V=0.94$, 2.56, and 2.16. Two values, $V=0.94$ [a SDW insulator, marked with a triangle in Fig. $\left.1, \Delta_{\text {charge }}\left(\phi_{\text {anti }}\right)=1.44\right]$ and $V=2.56$ [a CDW insulator, marked with an upside down triangle in Fig. $\left.1, \Delta_{\text {charge }}\left(\phi_{\text {anti }}\right)=1.36\right]$ are chosen such that $\chi_{\Xi}\left(\phi_{\text {anti }}\right)$ is the same. We also consider $V=2.16$ on the phase boundary between SDW and CDW with an almost diverging $\chi_{\Xi}\left(\phi_{\text {anti }}\right)$ (marked with a diamond in Fig. 1$)$. The latter case also has a vanishingly small charge gap but it should be considered as a strongly interacting metal.

\section{A. Weakly interacting system}

In Fig. 2 we show the current as function of time for a system with $U=1.5$ and $V=0.82$ for different electric field strengths. For illustrative purposes we start the analysis of the graph from the largest field, $F=0.4$, where it shows a regular damped $\mathrm{BO}$ in the time domain of interest. As we stated previously, $\chi_{\Xi}\left(\phi_{\text {anti }}\right)$ is largest at the anticrossings; thus the probability transfer from the ground state to excited states (also in analogy with LZ theory) is enhanced. Therefore at each anticrossing there is a high probability of transfer from a right-going wave $\left[-\frac{1}{L} \partial E_{n}(\phi) / \partial \phi>0\right]$ to another right-going wave. When the field is strong enough this transfer is very efficient such that the wave function, $|\psi(t)\rangle$, has a significant overlap with mainly one of the eigenstates of the instantaneous Hamiltonian. Finally when the maximum energy is reached, the wave function will start having significant overlap with leftgoing states and the current will change sign. This reflection for the high-field case happens exactly at $t=(2 F)^{-1}$.

To better understand the above description of the largefield response, we plot in Fig. 3 (a) the eigenstates of the instantaneous Hamiltonian as a function of time for a smaller system, with $L=6$ at half filling, for $F=0.4$ and the same 


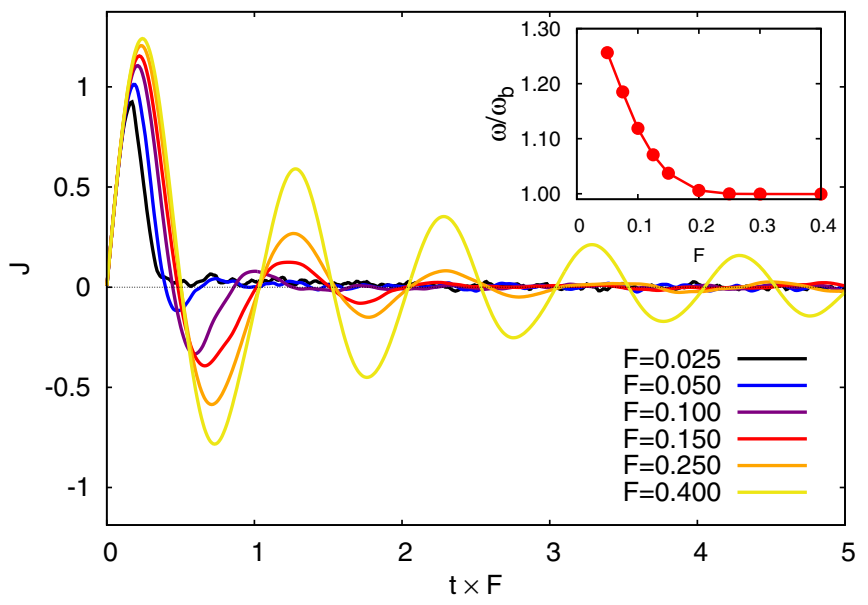

FIG. 2. (Color online) Current as a function of time for a ring with $U=1.5, V=0.82, L=10, N_{\uparrow}=N_{\downarrow}=5$, and for different electric field strengths. Inset: The frequency of the BO for different electric fields and the same parameters of the main graph with $\omega_{b}=F$.
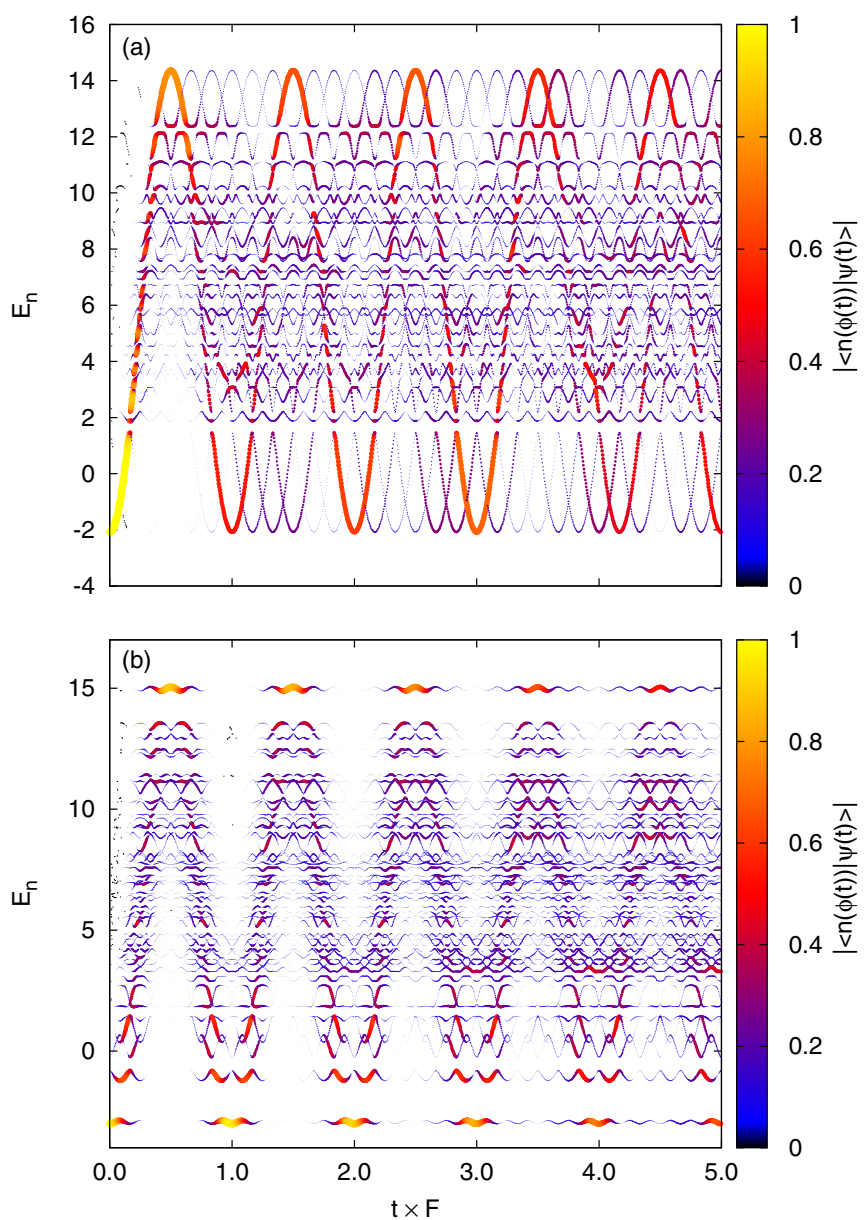

FIG. 3. (Color online) (a) Eigenvalues of the instantaneous Hamiltonian as function of time for a system with $L=6$ at half filling, $U=1.5, V=0.82, F=0.4$. The colors and the size of the points are given by the overlap of the time-dependent wave function with the instantaneous eigenstates, $|\langle n(\phi(t)) \mid \psi(t)\rangle|$. (b) The same as (a) but with a dimerization parameter $\eta=0.4$ and $F=4.0$. interaction parameters. Both the size of the points and their color code represent the magnitude of the overlap of the timedependent wave-function with the instantaneous eigenstates of $\hat{H}(\phi(t))$. Note that the spectrum is periodic with $2 \pi / L$; thus the first anticrossing happens at $t F=0.5 / L=0.0833$. This smaller ring shows similar behavior to the one presented in Fig. 2 when subjected to a strong field.

The formation of a coherent path for the probability transfer throughout the spectrum and the reflection at the topmost state when $t=(2 F)^{-1}$ can be clearly seen. However, a dissipative loss of the probability to both left-going and right-going waves is possible and the current becomes damped as a function of time. For higher fields the probability transfer is more efficient, which means that the damping of BO is suppressed.

Weak fields. Looking back to Fig. 2, the weakest field response, for $F=0.025$, is composed of two nonlinear effects. First, the state with high probability is reflected at smaller $t F$, well before it arrives at the other edge of the spectrum. This could be inferred from the fact that the current tends to change sign at smaller $t F$ than in the high-field case. Second, when the field is weak the probability transfer to excited states is smaller, which means that at each higher energy anticrossing there is a finite probability of remaining in the state with lower energy, which will contribute with a negative sign to the current of $|\psi(t)\rangle$. Therefore after an initial increase in current, the wave function will overlap with equally right-going and left-going instantaneous states and one ends up with a quasistationary regime in which the current is very small and fluctuates around zero.

We further elucidate this behavior by expressing the current as a function of the instantaneous eigenstates of $\hat{H}(\phi(t))$ :

$$
\begin{aligned}
\langle\hat{J}(t)\rangle= & \sum_{n} a_{n}(t)^{2}\langle n(\phi(t))|\hat{J}(\phi(t))| n(\phi(t))\rangle+\sum_{n \neq m} a_{n}(t) \\
& \times a_{m}(t) e^{i\left(\gamma_{n}(t)-\gamma_{m}(t)\right)}\langle m(\phi(t))|\hat{J}(\phi(t))| n(\phi(t))\rangle,
\end{aligned}
$$

where $a_{n}(t)=|\langle n(\phi(t)) \mid \psi(t)\rangle|$ describes the magnitude of the overlap of the time-dependent wave function with the instantaneous eigenstates and $\gamma_{n}(t)=\arg [\langle n(\phi(t)) \mid \psi(t)\rangle]$ describes the phases acquired by the wave function. We plot in Fig. 4(a), for $F=0.025$ and $L=8, a_{n}(t)^{2}$ as a function of the current for each eigenstate at time $t F=2.505$.
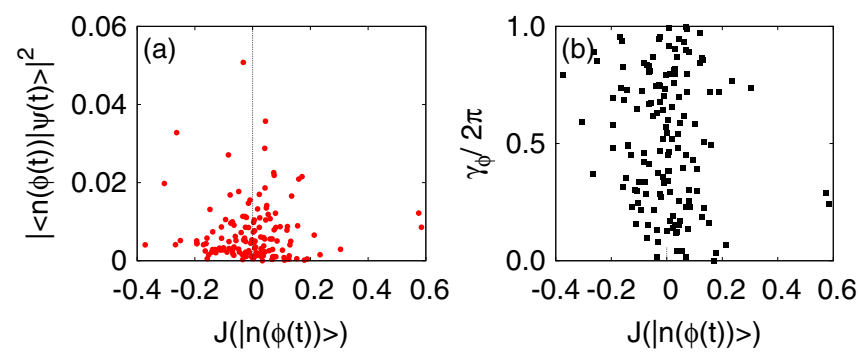

FIG. 4. (Color online) (a) $|\langle n(\phi(t)) \mid \psi(t)\rangle|^{2}$ for $L_{\text {sites }}=8$ and $N_{\uparrow}=N_{\downarrow}=4, U=1.5, V=0.82$, and $F=0.025$ at $t F=2.505$ as a function of the current of each state; $|n(\phi(t))\rangle$ are the eigenstates of the instantaneous Hamiltonian. (b) $\gamma_{n}=\arg [\langle n(\phi(t)) \mid \psi(t)\rangle]$ for the same parameters. 
Observe that the probability amplitudes as a function of current are approximately symmetrically distributed between left-going and right-going states; this in turn implies that the first term of Eq. (15), i.e., the diagonal expectation value of the current, becomes approximately equal to 0 . Moreover, the phases, $\gamma_{n}$, which are presented in Fig. 4(b), are distributed uniformly between 0 and $2 \pi$ therefore leading to the dephasing of nondiagonal terms in Eq. (15), and finally the total current is approximately equal to zero.

One should notice that for the case with $L=8$ the current is not completely equal to zero for the long-time response, but it acquires a small but finite value that fluctuates around zero, indicating the fact that the number of eigenstates that contribute is small due to finite-size effects. These fluctuations are suppressed for larger systems as we show in the following sections.

Intermediate fields. We next analyze the response to intermediate fields between the full dissipative case for $F=0.025$ and the full oscillating one for $F=0.4$. When the electric field strength is increased the reflection of the high-probability state gradually approaches the largest eigenstate of the spectrum. This could be clearly recognized in Fig. 2 where the time $t F$ for which the current changes its sign approaches 0.5 . At the same time the BO period, which is generally less than $F^{-1}$, gradually approaches $F^{-1}$. This is shown in the inset of Fig. 2 where we plot the frequency of $\mathrm{BO}$ as function of field strength. A similar behavior was also reported in metallic spinless systems subjected to a uniform electric field [38]. Our investigation should also be relevant for that case.

Here, we mention that similar to the electric breakdown case, where a mapping to a quantum random walk [54] on a semi-infinite chain was proposed, here the problem of $\mathrm{BO}$ damping also could be mapped to a quantum random walk but on a chain with two edge states. However, as we will present in the following, the actual long-time response to an electric field depends strongly on the probability transfer between subsequent states throughout the whole spectrum. It is therefore necessary to design a random walk for which the probability transfer is also randomized but taken from specific distributions, which could be chosen based on the level statistics of the Hamiltonian [55].

Dimerization. In Fig. 5, we show the current as a function of time for a system with the same interactions as in the metallic case but with a dimerization parameter $\eta=0.4$. We call this state a correlated band insulator (BI). The general arguments presented for the metallic case hold here; however there are also differences, which we explain in the following. As expected, dimerization induces the opening of a charge gap $\left[\Delta_{\text {charge }}\left(\phi_{\text {anti }}\right)=1.74\right]$ and the electric field breakdown is postponed to larger fields. Additionally, a dissipative regime appears only at $F=0.2$. At this field strength the breakdown has already happened and the instantaneous ground state has a very small contribution to $|\psi(t)\rangle$. For larger fields, i.e., $F=0.4$, first the current starts to show irregular oscillations, then at $F=0.6$ the current becomes oscillatory but with a frequency of the BO larger than $F$.

Finally, at even larger fields, $F=4.0$, the current is oscillatory with $\omega=F$. This is achieved for much larger fields than the ones presented for the weakly interacting metal, as shown in the inset of Fig. 5. The first notable

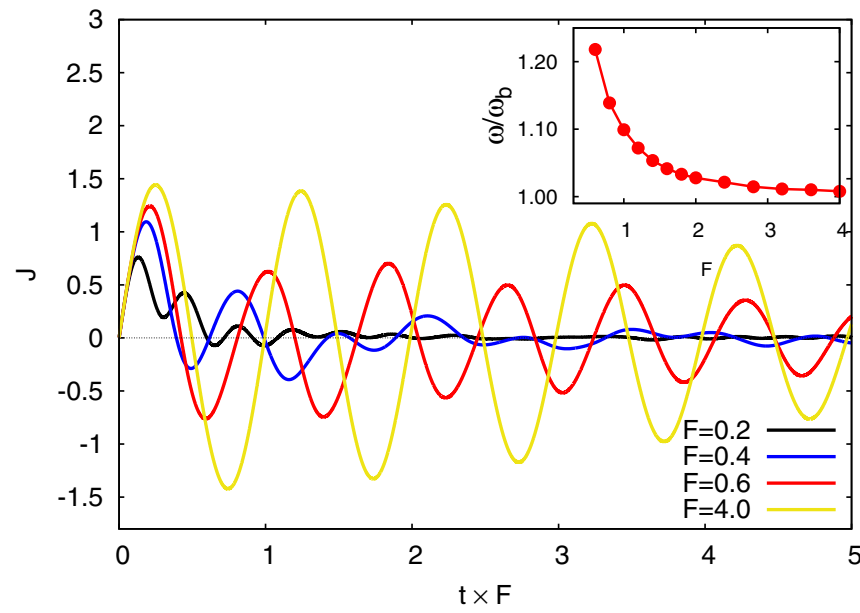

FIG. 5. (Color online) Current as function of time for a dimerized ring with $L_{\text {sites }}=10$ and $N_{\uparrow}=N_{\downarrow}=5, U=1.5, V=0.82, \eta=0.4$ (see the definition of the hopping parameter following Eq. (1)) and for different electric field strengths. The inset shows the frequency of the Bloch oscillations for different electric fields and the same parameters of the main graph with $\omega_{b}=F$.

difference between the metal and the correlated BI is that here BOs with larger frequencies survive for larger $t F$. This is different from the metallic case where BOs with larger frequencies are strongly damped. Furthermore, one may expect that the dimerization may only postpone the breakdown and the transition to the oscillatory behavior should not be affected as long as the dimerization only affects the low-energy part of the spectrum by opening up a ground-state charge gap. However, the presence of long-lasting BOs with a period less than $F^{-1}$ implies the presence of states with small $\chi_{\Xi}$ in the middle of the spectrum and which reflects back a high-probability state.

Roughly speaking, these states could be at the edge of a cluster of eigenstates, and are separated by a large gap from the next subsequent state and therefore play the role of an edge state. However, we emphasize that not only the gap but also the actual value of $\chi_{\Xi}$ of each eigenstate is an important factor that affects the nonadiabatic behavior of the system. In order to visualize again the overlap of the time-dependent wave function with the whole spectrum, we turn back to Fig. 3(b), where the overlap of $|\psi(t)\rangle$ with the instantaneous eigenstates of $\hat{H}(\phi(t))$ is plotted as function of time for a smaller dimerized system with $L=6, \eta=0.4$, and $F=4.0$. Again the smaller ring behaves the same as a larger system with $L=10$ when subjected to strong fields. As is clear from the plot the recurrences of the ground state and the state with largest energy occur periodically at times $t=F^{-1}$.

A noticeable feature of the propagation in dimerized systems is the fact that the overlap of $|\psi(t)\rangle$ with the instantaneous eigenstates is very nonlocal in the energy domain, meaning that the path of high-probability transition is broadened in comparison to the metallic system. Noticeably, the wave function starts to have finite overlap around the first anticrossing not only to the first excited state but also with the second excited state. Therefore, a two-level approximation (LZ-like) is not appropriate for breakdown of the ground state. 
The dimerization leads to a stronger insulator not only in the sense that it postpones the electric field breakdown, but it also largely affects the overlap of $|\psi(t)\rangle$ with states located in the middle of the spectrum. In short, while the breakdown and the appearance of the dissipative behavior depends mostly on the low-energy part of the spectrum, the transition from the dissipative to the oscillatory behavior largely depends on the clustering of eigenstates in the middle of the spectrum.

\section{B. Strongly interacting system}

For the cases with strong interactions, as stated before, we choose $U=4.0$ and three different nearest-neighbor interactions, $V=0.94$ (SDW insulator), $V=2.56$ (CDW insulator), and $V=2.16$ (metallic case). For the insulating cases we choose the interaction parameters such that both cases acquire the same ground state $\chi_{\Xi}\left(\phi_{\text {anti }}\right)$ as seen in Fig. 2.

We plot in Fig. 6(a) the current as a function of time for a very small electric field, i.e., $F=0.002$, for a ring of size $L=10$. Both insulating systems appear to be in the adiabatic regime, where the current shows an oscillatory behavior with a period equal to $1 / F L$. However, the metallic case shows oscillations with a doubled period, $2 / F L$. The main reason for this comes from the fact that for the metallic case the
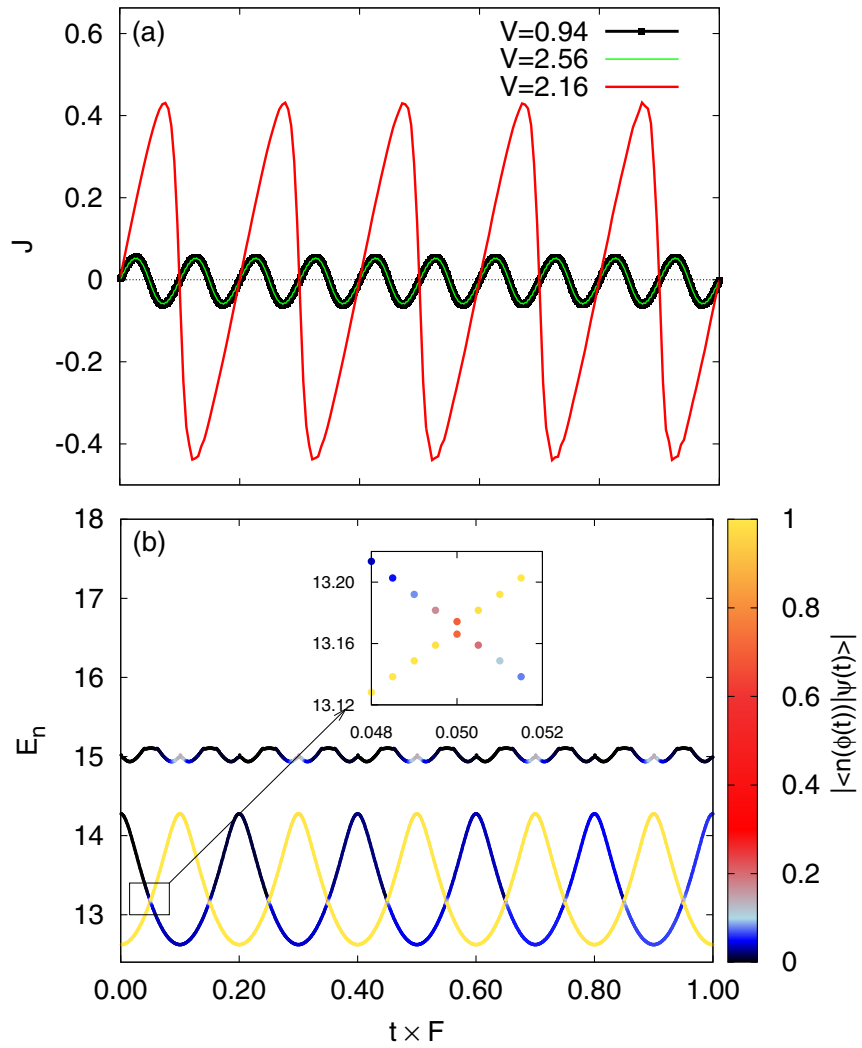

FIG. 6. (Color online) (a) Current as function of time for very small field $F=0.002$ for different interactions. (b) The energy dispersion of the first three excited states of the instantaneous Hamiltonian together with the overlap of these states with $|\psi(t)\rangle$ as function of time for $U=4.0, V=2.16$. The inset of panel (b) shows a zoom-in of the anticrossing region. Colors represent the overlap of the time-dependent wave function with the instantaneous eigenstates.

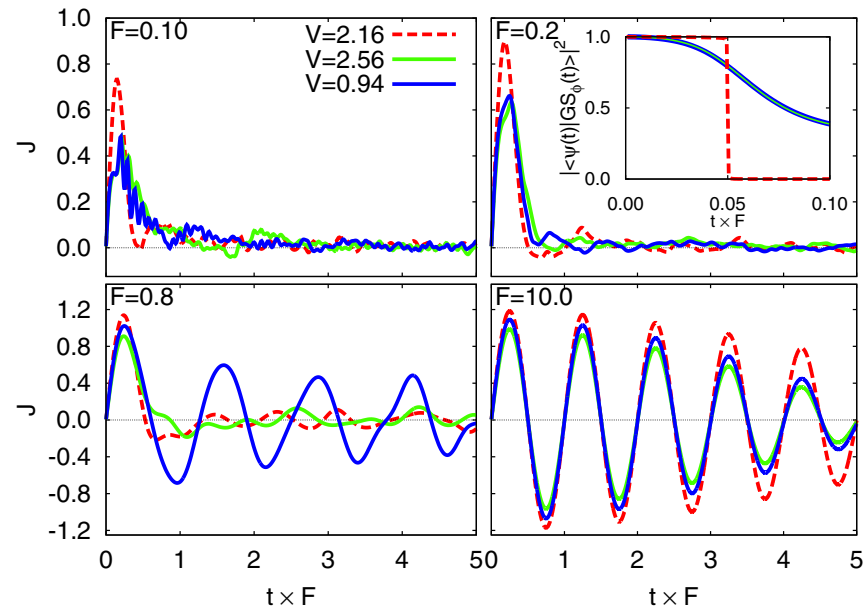

FIG. 7. (Color online) Current as a function of time for different interactions and different field strengths. The inset shows the square of the overlap of $|\psi(t)\rangle$ with the instantaneous ground state of $\hat{H}(\phi(t))$ for different interactions and $F=0.2$.

probability is transferred completely to the first excited state due to very large $\chi_{\Xi}\left(\phi_{\text {anti }}\right)$; i.e., it cannot be considered in the adiabatic regime even at these small fields. This is illustrated in Fig. 6(b), where the energies of the first three states of $\hat{H}(\phi(t))$ are shown as function of time (and implicitly as a function of flux), together with the overlap of $|\psi(t)\rangle$ with these three states.

As is obvious, because of the very large $\chi_{\Xi}\left(\phi_{\text {anti }}\right)$, there is a very large overlap with the first excited state after the first anticrossing; however the field is very small such that it cannot overcome the gap between the first excited and second excited state. $|\psi(t)\rangle$ only has an extremely small overlap with the second excited state, which leads to the fact that the probability is reflected back to the ground state and one ends up with current oscillations with a period twice the adiabatic expectation. The breakdown field is now achieved when the gap between the first and second excited states is overcome.

We next describe the response of strongly interacting systems to larger fields. In Fig. 7 we present the current as a function of time for different field strengths and for the three interaction choices introduced previously. For $F=0.1$ all the cases show a dissipative behavior; however the insulating ones show small peaks in the current before it arrives at the quasistationary zero-current state. The period of these peaks is approximately equal to $1 / F L$, which therefore implies that the overlap of $|\psi(t)\rangle$ with the instantaneous ground state does not vanish quickly and manifests itself as small peaks in the current. This is not the case for $V=2.16$ where the overlap with the ground state is lost immediately at the anticrossing (see the inset of Fig. 7 for $F=0.2$ ) and the current behaves smoothly from the beginning of the evolution. For stronger fields, $F=0.2$, the change of the current is large, such that the current fluctuations due to the finite overlap with the ground state disappear.

In the inset of Fig. 7 we show the square of the overlap of $|\psi(t)\rangle$ with the ground state of the instantaneous Hamiltonian. It is clear that for the two insulating cases for which we set $\chi_{\Xi}\left(\phi_{\text {anti }}\right)$ to be equal to each other $\left[\chi_{\Xi}\left(\phi_{\text {anti }}\right)\right.$ of SDW 


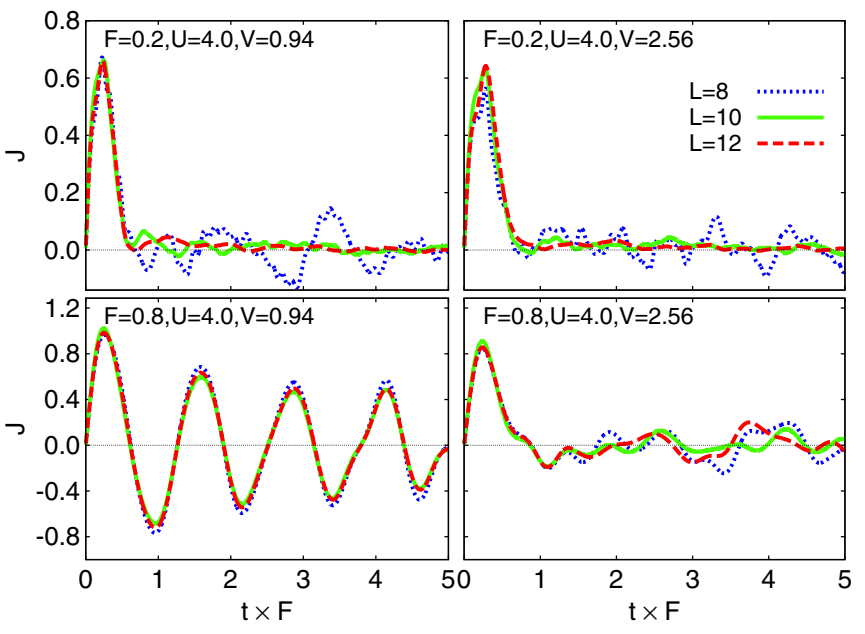

FIG. 8. (Color online) Current as a function of time for different interactions and different field strengths and different sizes of the system.

and CDW insulating systems], the decay of the ground state is identical. Notice, we observe some deviations between the ground-state decay of these two cases for smaller field strengths. Furthermore, in the dissipative cases for $F=0.1$ and $F=0.2$ both insulating cases behave in the same way even for larger times even though the interaction strengths are very different and one describes a SDW insulator while the other one is a CDW insulator with different excitation.

When the field is increased to $F=0.8$, the SDW insulator with $V=0.94$ starts to show oscillations with large amplitude. On the other hand the metallic and CDW cases are still near the dissipative regime with a small long-time current, but irregular. For even larger fields, $F \simeq 1.6$ (not shown here), all three cases show oscillations with large amplitude but which are irregular. It is only when the strength of the electric field is very large, $F=10.0$, that all the cases show regular $\mathrm{BO}$ as shown in Fig. 7.

Finite-size effect. To see the effect of the size of the system on the transition from a dissipative to an oscillatory pattern, we plot in Fig. 8 the current as a function of time for different sizes for $V=0.94$ (SDW insulator) and $V=2.56$ (CDW insulator). We observe that for all cases the fluctuations of the current in the dissipative regime $(F=0.2)$ are suppressed for larger sizes. This is due to the fact that $|\psi(t)\rangle$ acquires overlap with a much larger number of states when the size is increased. This implies that a more efficient dephasing of the current is achieved [see the discussion following Eq. (15)]. However, in the strong-field regime, once the transition to oscillatory behavior occurs, the size effect is negligible, showing that the sizes of the gaps in the middle of the spectrum do not depend strongly on the size, at least not for the strong interactions considered here.

Order parameters. In Fig. 9, we show the SDW and CDW order parameters as functions of time for the two insulating cases. As is clear from Fig. 9(b) for the SDW ordered system $(V=0.94), O_{\text {SDW }}$ only drops gradually as function of time; however at the same time $O_{\mathrm{CDW}}$ is enhanced at the beginning of the evolution [see Fig. 9(a)]. This further implies the presence of a CDW state near the bottom of the spectrum [23].
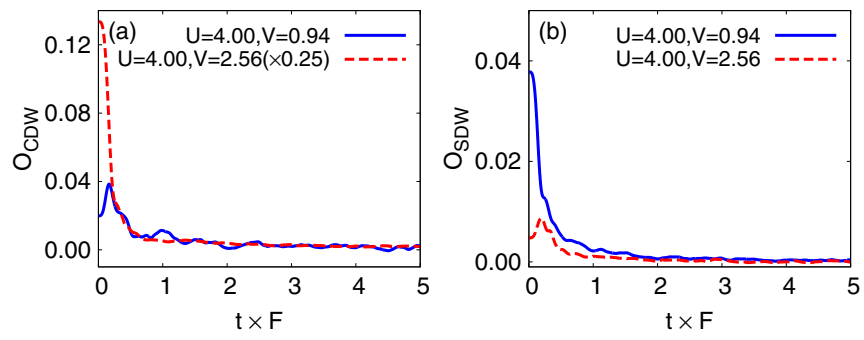

FIG. 9. (Color online) (a) CDW order parameter as a function of time for a system with $L=12$ at half filling derived with $F=0.2$. (b) SDW order parameter as function of time and the same parameters as in plot (a).

Finally, at longer times both order parameters dissipate during the evolution arriving at a quasistationary state with almost vanishing value for larger times. The CDW ordered system shows a similar behavior but with reversed $O_{\mathrm{CDW}}$ and $O_{\mathrm{SDW}}$ contributions [see Figs. 9(a) and 9(b)]. Therefore, the transient regime shows that since the two order parameters are in competition, the mechanism of destroying the dominant order is the proliferation of the competing one.

\section{CONCLUSIONS}

In conclusion we investigated the nonlinear response of a closed interacting fermionic system as modeled by an extended Hubbard model. A weakly interacting metallic system at the boundary of SDW-CDW shows a dissipative behavior for low fields. The main reason for this is the fact that $|\psi(t)\rangle$ acquires overlap with a large number of left-going and right-going states at large times. This in turn implies that the quasistationary state acquires zero current. Bloch oscillations start smoothly with a frequency larger than $F$. The main reason for this is the fact that the reflection happens at the lower part of the spectrum, thus effectively decreasing the bandwidth. Upon increasing the field strength the probability transfer at each anticrossing is more efficient. This leads to a more regular recurrence of the ground state and the topmost excited state with period $F^{-1}$, which can be seen from the oscillations in the current.

Upon dimerization of the metallic system, the formation of the dissipative regime is postponed to larger fields due to the formation of a charge gap. However, the main difference between the dimer case and the metallic system resides in the fact that, first, it shows irregular current oscillations before they turn into regular BOs and second, the BOs with larger frequencies survive for larger $t F$. In analogy with the metallic case subjected to strong field, this implies the existence of states at the middle of the spectrum with low $\chi_{E}(\phi)$ (or roughly speaking the formation of large midgaps in the relevant excitations) that play the role of a band-edge state and reflect back the overlap probability at the middle part of the spectrum even for large electric fields. Finally, the dimerized system also shows regular BOs with frequency equal to $F$ for large enough electric fields. The value for which the dimerized system shows regular BOs is much larger than those found for the metallic system even though the interactions are identical.

For stronger interacting systems when the interactions are chosen such that the ground-state $\chi_{\Xi}\left(\phi_{\text {anti }}\right)$ is the same for both cases, then the ground-state decay for both CDW insulator 
and SDW insulator behaves exactly the same for short times. This similarity of the ground-state decay manifests itself even for larger times and for both low- and high-field dissipative regimes. However significant differences arise for large-time response between the two cases for large electric fields. While SDW shows oscillatory behavior with large magnitude, the CDW insulator and the strong interacting metallic system only exhibit irregularities with small oscillations. Different from the weakly interacting metallic system and the dimer case, in the strongly interacting regime these irregularities are extended to intermediate fields and only for very large fields, $F=10.0$, regular BO with a period of $F^{-1}$ is observed. This effect appears to be little affected by size, since the SDW and CDW insulators, for $L=10$ and $L=12$, show the same qualitative and even quantitative behavior. This implies that the reorganization of the spectrum is affected much more by the interaction than by the finite-size-induced discreteness.

\section{ACKNOWLEDGMENTS}

This work was supported by the Flemish Science Foundation (Fonds Wetenschappelijk Onderzoek - FWO) and the Methusalem program of the Flemish government. One of us (L.C.) receives support as a postdoctoral fellow of the FWO.
[1] A. Polkovnikov, K. Sengupta, A. Silva, and M. Vengalattore, Rev. Mod. Phys. 83, 863 (2011).

[2] R. Chen and H. Guo, Comput. Phys. Commun. 119, 19 (1999).

[3] A. Weisse and H. Fehske, Lect. Notes Phys. 739, 545 (2008).

[4] M. Hochbruck and C. Lubich, SIAM J. Numer. Anal. 34, 1911 (1997).

[5] F. Verstraete, J. J. García-Ripoll, and J. I. Cirac, Phys. Rev. Lett. 93, 207204 (2004).

[6] J. K. Freericks, Phys. Rev. B 77, 075109 (2008).

[7] M. Eckstein and P. Werner, Phys. Rev. B 88, 075135 (2013).

[8] F. B. Anders and A. Schiller, Phys. Rev. Lett. 95, 196801 (2005).

[9] A. Hackl and S. Kehrein, Phys. Rev. B 78, 092303 (2008).

[10] T. L. Schmidt, P. Werner, L. Mühlbacher, and A. Komnik, Phys. Rev. B 78, 235110 (2008).

[11] H. Schoeller and F. Reininghaus, Phys. Rev. B 80, 045117 (2009).

[12] F. Andraschko and J. Sirker, Phys. Rev. B 89, 125120 (2014).

[13] M. Eckstein, M. Kollar, and P. Werner, Phys. Rev. B 81, 115131 (2010).

[14] M. Eckstein, A. Hackl, S. Kehrein, M. Kollar, M. Moeckel, P. Werner, and F. A. Wolf, Eur. Phys. J. Spec. Top. 180, 217 (2009).

[15] C. Kollath, A. M. Lauchli, and E. Altman, Phys. Rev. Lett. 98, 180601 (2007).

[16] S. Genway, A. F. Ho, and D. K. K. Lee, Phys. Rev. A 86, 023609 (2012).

[17] M. Rigol and L. F. Santos, Phys. Rev. A 82, 011604 (2010).

[18] K. A. Al-Hassanieh, F. A. Reboredo, A. E. Feiguin, I. Gonzalez, and E. Dagotto, Phys. Rev. Lett. 100, 166403 (2008).

[19] M. Mierzejewski, J. Bonča, and P. Prelovšek, Phys. Rev. Lett. 107, 126601 (2011)

[20] M. Mierzejewski, L. Vidmar, J. Bonča, and P. Prelovšek, Phys. Rev. Lett. 106, 196401 (2011).

[21] M. Eckstein and P. Werner, Phys. Rev. Lett. 107, 186406 (2011).

[22] M. Eckstein, T. Oka, and P. Werner, Phys. Rev. Lett. 105, 146404 (2010).

[23] H. Lu, S. Sota, H. Matsueda, J. Bonča, and T. Tohyama, Phys. Rev. Lett. 109, 197401 (2012).

[24] A. Takahashi, H. Itoh, and M. Aihara, Phys. Rev. B 77, 205105 (2008).

[25] M. Mierzejewski, J. Luczka, and J. Dajka, J. Phys.: Condens. Matter 22, 245301 (2010).

[26] F. Heidrich-Meisner, I. González, K. A. Al-Hassanieh, A. E. Feiguin, M. J. Rozenberg, and E. Dagotto, Phys. Rev. B 82, 205110 (2010).

[27] T. Oka, R. Arita, and H. Aoki, Phys. Rev. Lett. 91, 066406 (2003).
[28] T. Oka and H. Aoki, Phys. Rev. Lett. 95, 137601 (2005).

[29] L. D. Landau, Phys. Z. Sowjetunion 2, 46 (1932).

[30] C. Zener, Proc R. Soc. A 137, 696 (1932).

[31] Z. Lenarčič and P. Prelovšek, Phys. Rev. Lett. 108, 196401 (2012).

[32] W.-L. You, Y.-W. Li, and S.-J. Gu, Phys. Rev. E 76, 022101 (2007).

[33] J. Sirker, Phys. Rev. Lett. 105, 117203 (2010).

[34] J. Feldmann, K. Leo, J. Shah, D. A. B. Miller, J. E. Cunningham, T. Meier, G. von Plessen, A. Schulze, P. Thomas, and S. SchmittRink, Phys. Rev. B 46, 7252 (1992).

[35] K. Leo, P. H. Bolivar, F. Bruggemann, R. Schwedler, and K. Kohler, Solid State Commun. 84, 943 (1992).

[36] C. Waschke, H. G. Roskos, R. Schwedler, K. Leo, H. Kurz, and K. Kohler, Phys. Rev. Lett. 70, 3319 (1993).

[37] P. Voisin, J. Bleuse, C. Bouche, S. Gaillard, C. Alibert, and A. Regreny, Phys. Rev. Lett. 61, 1639 (1988).

[38] M. Mierzejewski and P. Prelovšek, Phys. Rev. Lett. 105, 186405 (2010).

[39] L. Vidmar, J. Bonča, M. Mierzejewski, P. Prelovšek, and S. A. Trugman, Phys. Rev. B 83, 134301 (2011).

[40] F. Sawano, I. Terasaki, H. Mori, T. Mori, M. Watanabe, N. Ikeda, Y. Nogami, and Y. Noda, Nature (London) 437, 522 (2005).

[41] Y. Taguchi, T. Matsumoto, and Y. Tokura, Phys. Rev. B 62, 7015 (2000).

[42] S. Iwai, M. Ono, A. Maeda, H. Matsuzaki, H. Kishida, H Okamoto, and Y. Tokura, Phys. Rev. Lett. 91, 057401 (2003).

[43] H. Okamoto, H. Matsuzaki, T. Wakabayashi, Y. Takahashi, and T. Hasegawa, Phys. Rev. Lett. 98, 037401 (2007).

[44] M. Greiner, O. Mandel, T. W. Hansch, and I. Bloch, Nature (London) 419, 51 (2002).

[45] M. Köhl, H. Moritz, T. Stöferle, K. Günter, and T. Esslinger, Phys. Rev. Lett. 94, 080403 (2005).

[46] I. Bloch, Nat. Phys. 1, 23 (2005).

[47] T. Esslinger, Annu. Rev. Condens. Matter Phys. 1, 129 (2010).

[48] M. V. Berry, Proc. R. Soc. A 392, 45 (1984).

[49] P. Zanardi and N. Paunković, Phys. Rev. E 74, 031123 (2006).

[50] S. Greschner, A. K. Kolezhuk, and T. Vekua, Phys. Rev. B 88, 195101 (2013).

[51] C. Lanczos, J. Res. Nat. Bur. Stand. 49, 255 (1950).

[52] A. Weisse and H. Fehske, Lect. Notes Phys. 739, 529 (2008).

[53] S. Ejima and S. Nishimoto, Phys. Rev. Lett. 99, 216403 (2007).

[54] T. Oka, N. Konno, R. Arita, and H. Aoki, Phys. Rev. Lett. 94, 100602 (2005)

[55] D. Poilblanc, T. Ziman, J. Bellissard, F. Mila, and G. Montambaux, Europhys. Lett. 22, 537 (1993). 